\section{Pneumoperikard und gedeckte Aortenruptur bei Thoraxkontusion}

Verletzungen des Perikards mit begleitender Aortenverletzung sind selten (Clark et al., Surgery 1983; 93: 495). Sie treten in der Regel bei Verkehrsunfällen infolge abrupter Dezeleration auf. Es wird über einen Patienten berichtet, der sich eine Verletzung dieses Musters bei einem PKW-Frontalzusammenstoß zuzog.

\section{Fallbeschreibung}

An der Unfallstelle fand der Notarzt einen 31jährigen, auf dem Fahrersitz eingeklemmten Patienten vor. Nach Bergung und Hubschrauberverlegung in die unfallchirurgische Notaufnahme zeigte die kranielle Computertomographie ein Schädel-Hirn-Trauma III. ${ }^{\circ}$ mit temporobasalen Kontusionsblutungen und Hirnödem. Die thorakoabdominale Spiral-CT ergab rechtsseitige Rippenserienfrakturen, eine Lungenkontusion mit beidseitigem rechtsbetonten Hämatothorax (Abb.1) trotz einliegender Thoraxdrainagen, ein Pneumoperikard (Abb. 2) und ein Mediastinalhämatom mit gedeckter Ruptur der Aorta descendens (Typ B Dissektion, Abb. 3 und 4). Abdominal fand sich eine ausgedehnte Leber- und Milzlazeration.

In der unmittelbar anschließenden Laparatomie konnte nach Splenektomie sowie Naht und Packing der Leberruptur der massive intraabdominelle Blutverlust beherrscht werden. Bei persistierender hämodynamischer Instabilität mußte die Arteria gastrica dextra ligiert sowie ein plötzlicher Kreislaufstillstand durch mechanische und chemische Reanimation intraoperativ behandelt werden. Das Pneumoperikard wurde postoperativ auf der chirurgischen Intensivstation punktiert und drainiert.

Die kardiochirurgische Versorgung der gedeckten Aortenruptur war aufgrund des hohen Katecholaminbedarfs und der Beatmungspflichtigkeit $\left(\mathrm{FiO}_{2}=1\right)$ nicht unmittelbar möglich. Zwanzig Stunden nach Einlieferung kam der Patient infolge transtentorieller Herniation ad exitum.

Fortschr Röntgenstr 2000; 172: 561 -562 (c) Georg Thieme Verlag Stuttgart · New York ISSN 1438-9029

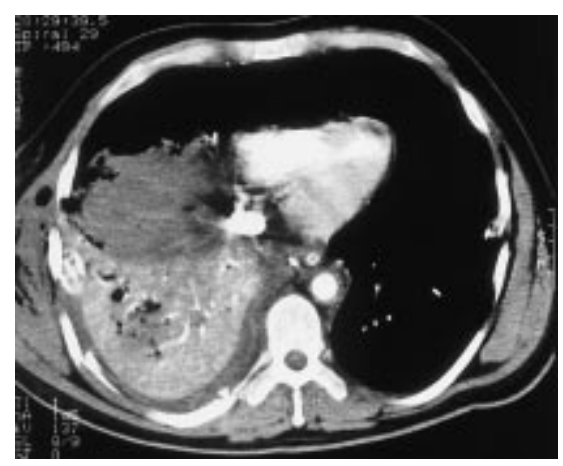

Abb. 1 Kontrastmittelverstärkte Spiral-CT des Thorax: Lungenkontusion rechts mit Einblutung im Unterlappen, beidseitige Thoraxdrainage bei Hämatopneumothorax rechts und Hämatom im hinteren Mediastinum bei Rippenfrakturen. Thoraxwandemphysem rechts. Auffällig ist die Distanzierung der Herzkontur von der Retrosternalfläche.

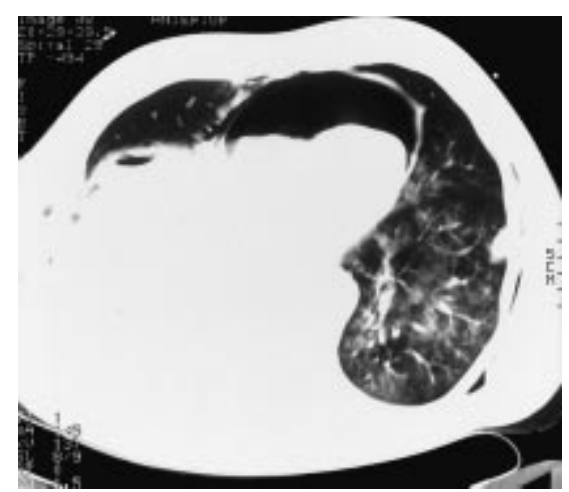

Abb. 2 Identische Aufnahmeposition wie in Abb. 1 zur Darstellung des raumfordernden Pneumoperikards. Linksseitig Lungenkontusionsareale.

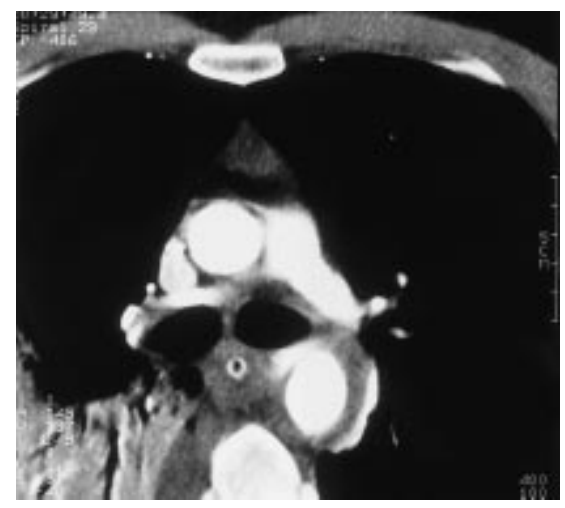

Abb. 3 Infrakranialer Axialschnitt mit Darstellung des Kontrastmittelparavasats und gedeckter Ruptur bei Dissektion der Aorta descendens thoracalis. Mediastinalhämatom.

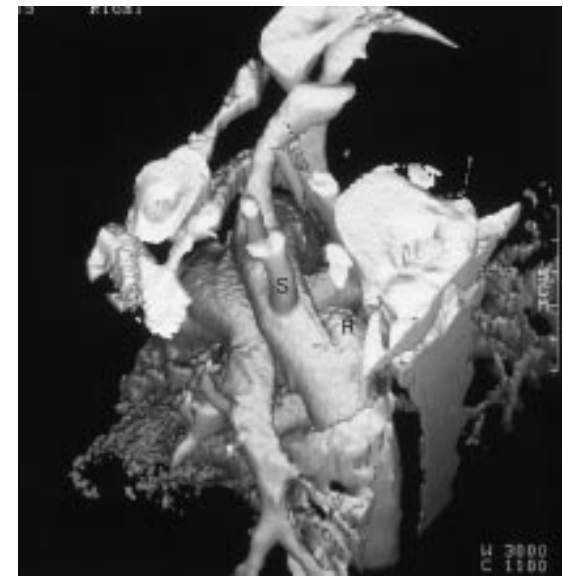

Abb. 4 Oberflächenrekonstruktion aus dem mit $1 \mathrm{~mm}$ Inkrement nachberechneten SpiralDatensatz. Darstellung der nach dorsal und lateral gerichteten, im hinteren Mediastinum gelegenen gedeckten Ruptur (R) der Aorta descendens thoracalis distal des Abgangs der A. subclavia sinistra (S).

Autoptisch bestätigte sich eine $3,5 \mathrm{~cm}$ lange, quer verlaufende, gedeckte Ruptur am Übergang des Arcus aortae zur Aorta descendens distal des Abgangs der Arteria subclavia bis in das adventitielle Bindegewebe. Im Perikard und im Mediastinum befanden sich zum Obduktionszeitpunkt $80 \mathrm{ml}$ hämorrhagische Flüssigkeit. Intrakraniell waren bei Schädelbasisfraktur beidseitig epidurale Hämatome, temporale zerebrale Kontusionsherde und ein Hirnödem nachweisbar.

\section{Diskussion}

Nach einer Studie von Chan et al. (Chan et al., Eur J Radiol 1996; 23: 23) sind 95\% aller polytraumatisierten Patienten in Verkehrsunfälle verwickelt, bei denen in 54\% Personenkraftwagen beteiligt sind. Verletzungen des Brustkorbs treten hierbei mit $60 \%$ etwa zwei- bis dreimal so häufig auf wie viszerale Verletzungen, so daß insbesondere bei Dezelerationstraumata an eine Koinzidenz von Verletzungen der Aorta und des Herzens zu denken ist. Bei den unter 40jährigen polytraumatisierten Unfallopfern sind Thoraxverletzungen in über $50 \%$ der Fälle für den Tod verantwortlich. Bereits 1939 wiesen Crynes und Hunter Einrisse des Perikards bei Thoraxverletzungen nach (Crynes et al., Arch Intern Med 1939; 64: 719). Bei 4107 an ihrem Institut durchgeführten Obduktionen konnten sie in 22 Fällen $(0,53 \%)$ traumatische Perikardeinrisse nachweisen. 
Das für ein Pneumoperikard ursächliche Verletzungsmuster ist sehr variabel und reicht von millimetergroßen Einrissen bis zur kompletten pleuroperikardialen Zerreißung. Clark et al. faßten 1983 die Ergebnisse von 142 publizierten Fällen mit traumatischer Perikardruptur für den Zeitraum von 1939 bis 1982 zusammen (Clark et al., Surgery 1983; 93: 495). In etwa der Hälfte der Fälle finden sich die Perikardeinrisse mit dem N. phrenicus linksseitig, in etwa $17 \%$ sind die pleuroperikardialen Einrisse rechtsseitig. Eine Zwerchfellruptur läuft selten bis in das Perikard. Einrisse im vorderen Mediastinum oder der Rückseite des Herzbeutels stellen eine Rarität dar. In nur vier Fällen $(2,8 \%)$ fand sich eine begleitende Verletzung der Aorta, wobei das hier berichtete Pneumoperikard mit $6 \%$ nur geringfügig häufiger gefunden wurde. Infolge einer mediastinalen oder abdominalen Verbindung ist ein Spannungspneumoperikard mit konsekutiver Perikardtamponade insbesondere bei beatmeten polytraumatisierten Patienten als weitere Komplikation bekannt (Nakamura et al., Nippon Kyobu Geka Gakkai Zasshi 1994; 42: 1242). In der Literatur werden Tracheal- oder Karinaeinrisse beschrieben (Chan et al., Eur J Radiol 1996; 23: 23). Obwohl bei der Obduktion des Patienten nicht explizit ein Perikardriß beschrieben wurde, ist in der Synopsis aller Befunde eine traumatische Fistel zwischen Trachea oder Pleura und Herzbeutel wahrscheinlich.

Die Spiral-Computertomographie ist in der Notfalldiagnostik unverzichtbar (Wicky et al., Eur Radiol 1998; 8: 828). Die Darstellung von Aortenrupturen oder -dissektionen in der Spiral-CT ermöglicht die exakte Beurteilung der Lokalisation und Ausdehnung einschließlich der Beteiligung supraaortaler Gefäße. Im Gegensatz zur Aortographie ermöglicht die Spiral-CT mit mehrdimensionaler Bildnachverarbeitung eine schnelle und sichere Diagnostik. Bei 1518 Patienten mit stumpfer Thoraxverletzung wurden Aortenverletzungen mittels Spiral-CT mit einer Sensitivität von $100 \%$ bei einer Spezifität von $82 \%$ nachgewiesen (Gavant et al., Radiology 1995; 125). Beim instabilen, polytraumatisierten Patienten ist die Spiral-CT das Diagnostikum der ersten Wahl.

R. Leppek, B. Kusch, E. Brück, Marburg 